\title{
COVID-19 Lockdown in India: An Experimental Study on Promoting Mental Wellness Using a Chatbot during the Coronavirus
}

\author{
V. P. Harshini Raji and P. Uma Maheswari \\ College of Engineering Guindy, Anna University, Chennai, 600025, India \\ ${ }^{*}$ Corresponding Author: V. P. Harshini Raji. Email: hello@harshiniraji.in \\ Received: 30 July 2020 Accepted: 12 December 2020
}

\begin{abstract}
India imposed the largest lockdown in the world in response to fight the spread of the Novel Coronavirus disease (COVID-19) from 19 March till 31 May 2020. The onset of the pandemic left the general public feeling psychosocially distressed, helpless, and anxious. The researcher developed a Messenger supported Chatbot, based on the broaden and build model, to cater to the healthy general public to promote positivity and mental well-being. 31 participants between 22 and 45 years old consensually took a pre-test, Chatbot intervention, and post-test. The Chatbot provided guided activities out of which positive affirmations, meditation, and exercises were mostly used. The qualitative data from the study shows that the majority of the participants strongly feel positivity is within themselves and that the tool provided a self-help approach to be me well, mentally during the lockdown. The intervention helped significantly reducing symptoms of psychosocial distress in six of the individual's postchatbot interventions. Participants' impressions of the tool suggest more preponderant opportunities for future research in technology-driven mental health support.
\end{abstract}

\section{KEYWORDS}

Mental health; COVID-19; chatbot; intervention study

\section{Introduction}

In the year 2020, an ongoing global pandemic enveloped the world, leaving millions sick and many dead. The novel coronavirus disease, COVID-19, also called the 2019-nCoV is said to have originated from a single source in December 2019 [1]. On 07 January 2020, the Chinese Center for Disease Control and Prevention (CCDC) identified and isolated this novel coronavirus named severe acute respiratory syndrome coronavirus 2 (SARSCoV-2). The SARSCoV-2 was transmitted between people who had close contacts with someone who was affected. Infected people may develop severe and even fatal respiratory diseases (e.g., acute respiratory distress syndrome (ARDS) and acute respiratory failure) which will lead to being treated under intensive care units [2].

The virus spread all through the world, gradually affecting almost all countries [3]. On January 30, 2020, the World Health Organization (WHO) declared the outbreak a Public Health Emergency of International Concern (PHEIC) (World Health Organization, January 31, 2020). WHO urged all countries to take strict measures to contain the spread of the virus and international flights were completely halted across all countries. Steps were taken keeping in mind the SARS outbreak of 2003 (World Health Organization, 2003). 
Although COVID-19 infects people of all ages, studies as of now, state that it puts people who are over 60 years old and people with underlying medical conditions like cardiovascular diseases, diabetes, chronic respiratory diseases, and cancer are at a higher risk of contracting the disease.

The outbreak of COVID-19 has caused tremendous psychological problems in different subpopulations [4]. The lockdowns and social distancing measures made it difficult for people to seek mental health support in person, hence, online mental health services flourished in this time around the world. Hotlines and mobile applications were promoted to help people in emergency psychological crisis management. Mental health professionals were providing individual counselling through online platforms like Alihealth and HaoDaiFu and provided other strategies to cope up with stress through social networks like WeChat and Weibo in China [5]. Hotlines were set up by the NHC to look after the psychological crisis intervention and notices were issued by the National Health Commission of China [6]. The Bureau of Disease Prevention and Control brought out the 'Guidelines for Psychological Assistance Hotlines during the New Coronavirus Pneumonia Outbreak' on the 7th February 2020, in alliance with the State Council Joint Prevention and Control Team to respond to the New Coronavirus Pneumonia, 2020 [7]. In India, apart from mobile phone applications like 'Arogya Setu', online websites that provide the latest COVID-19 related statistics, there were insignificant numbers of mental health-related solutions offered by the Government for individuals during the first lockdown.

In India, there has been limited attention paid to mental healthcare; during this crisis hour, basic social needs like transportation for migrant workers, food, and ration supply are needed, more than psychosocial remedies. A few Governmental, Non-Governmental Organizations (NGOs), private mental health clinics and practitioners have established hotlines, telepsychiatry, and e-mental health services to help the general public deal with the stressors. Private mental health professionals (MHPs) gathered in their interest to offer services online, set up their social media platforms to address psychosocial needs immediately after the world's largest lockdown, which was in India, was imposed on 24 March 2020. Many of the MHPs created their strategies, guidelines, and interventions through telephone and a newer medium-digital media.

Although during the beginning of the pandemic, there were not many online platforms where the individuals not affected by the coronavirus could receive mental health support, with time, around the mid of April 2020, the Ministry of Health and Family Welfare set up a website extension with resources to tackle mental illness. Audio-visual contents that can guide individuals to deal with smoking or drinking issues, mental health in children and migrant workers, dealing with the stigma associated with COVID-19, video content on Yoga, and stress management were displayed.

However, many private and semi-governmental NGOs like Sangath, Pushpagiri Insititute of Medical Sciences, NPTEL, etc., provided online webinars and lectures to help guide both psychiatrists and individuals to deal with the mental issues that may arise during this period. Apart from this, privately practicing psychologists and psychiatrists took to their social media handles to dissipate messages of keeping mentally well and took questions and answered them for their followers and listeners in online live sessions. iCall is a free psychosocial helpline that is part of the Tata Institute of Social Sciences, a deemed university in Mumbai. They had set up a separate COVID-19 helpline to tackle calls with issues like domestic violence, support for the marginalized communities like the LGBTQ, and stranded migrants.

The pandemic has created a huge disruption of the existing ways mental health issues are addressed across nations. COVID-19 has posed challenges for both caregivers and people affected with mental illness. However, with the immense growth in technology, new media platforms like social media, AIpowered software have enabled digital interactions that help with addressing the gap of physical helpseeking. This qualitative research paper illustrates with an experimental study, how a Chatbot that uses a psychological model called the broaden-and-build model can promote better mental health for people 
who are not affected by the coronavirus and are social distancing during a complete lockdown period like the one India is currently experiencing.

\section{Literature Review}

\subsection{Mental Health during a Pandemic}

Ramalingaswami [8] writes that the bubonic plague spread of 1994 triggered 600,000 people to leave the city of Surat in India within one night by all means- ox, carts, horses, or even by foot. Hordes of people moved out of the location in mere fear of the plague. The impact of the fear was far worse than the spread of the plague in itself. Fear comes with multiple psychological issues including panic, depression, anxiety, insomnia, and so on. Manderscheid [9] explains that there is a general rule of thumb- one-third of the population in an affected region will remain unaffected by the threat, one third will remain hypervigilant thereby leading to the growth in mental health issues such as insomnia, anxiety, and fear, and about the spread. One third will be completely left immobilized leading to depression and post-traumatic stress disorders (PTSD). Although this is just a general idea of what to expect in the case of a pandemic, the numbers might change keeping in mind the time, location, the kind of disease, and the methods used to measure the mental hazard.

During the Severe acute respiratory syndrome (SARS) outbreak in 2003, it was identified that there were two psychological phases-one being the initial phase and the latter, the repair phase. People felt a multitude of emotions such as vulnerability, uncertainty including cognitive symptoms of anxiety in the first phase. The second phase- the repair phase included feelings of depression and avoidance. Having clear directives, feeling protected, and being well equipped to face the outbreak, having some form of support or someone to speak with were some suggestions provided in research [10].

It is suggested by Sim et al. [11] that the response system for an epidemic by any nation should consider a psychological surveillance system. The system should include healthcare workers and their families and members of the community as well. The system must remain longitudinal to find out what triggers psychological distress and the positive points of coping strategies in place as well. Ursano [12] suggests that mental healthcare planning is vital in terms of a response system to epidemics and pandemics. If left unplanned for, the calamity of a psychological crisis can lead to a larger catastrophe. Planning properly ahead of the onset of an epidemic or pandemic only helps in healthcare relief and quicker recovery of people.

Giving another angle to the psychosocial distress caused by pandemics, Gao et al. [13] suggest that there is a high prevalence of mental health problems, which is positively associated with the higher social media exposure during the COVID-19 outbreak, by people who were self-quarantining. These findings illustrate that the government needs to pay more attention to mental health problems like depression, anxiety, and social distress among the public and help them deal with the 'infodemic'-the spread of extensive information pertaining to the virus and disease, that spreads like wildfire during any public health emergency, especially with the prevalence of social media.

During the SARS spread of 2003, there were no many online platforms that could be utilized for coverage of information regarding the spread nor provide mental health guidelines. But the emergence of $5 \mathrm{G}$ in the present times has enabled health professionals and authorities to provide services to patients through online platforms thereby reducing the time taken to transfer information and also inhibiting the need for people to step out of their homes and seek help for mental health issues [14,15].

Psychosocial disorders including depression, anxiety, stress increased with the increase in the number of confirmed cases and deaths [16] around the world. In China, online media were promoting mental wellness, like 'Public Psychological Self-Help Guideline for Pneumonitis with New Coronavirus Infection' [17], the series of 'New Coronavirus Science Popularization Knowledge', 'Online Lessons of Psychological Intervention during the New Coronavirus Pneumonia Outbreak' [18], and videos on 'Psychological 
Responses in the Fight Against New Crown Pneumonia' [19], 'Psychological Adjustment Guidelines for Coping with the New Coronavirus Pneumonia' were published [20]. Online mental health education through communication programs, such as WeChat, Weibo, and TikTok, has been widely used during the outbreak for medical staff and the public. Online self-help intervention systems using cognitive behavioral therapy (CBT) and artificial intelligence (AI) for treating depression, anxiety, and insomnia were also developed and promoted. 'Tree Holes Rescue' was a program that was developed to identify individuals at the risk of committing suicide by analyzing the status updates and messages the individual posts in their social media platform Weibo which will alert mental health volunteers and trigger them to help the individual [21]. Available literature about technology and mental health support during the COVID-19 pandemic provided in other countries remains minimal and is still being reviewed.

Currently, there are over four sixty million internet users in India, making India the 2nd largest online market ranked only behind China. Internet penetrations are constantly changing, in urban India, it increased from $60.6 \%$ in 2016 to $64.8 \%$ in 2017 . In a country like India where one in three people have access to a telephone, mental health care is becoming more accessible than before. The younger generation is moving to smartphones and online internet-based messaging, indicating that these platforms could be better utilized to provide mental healthcare, especially in a pandemic situation.

\subsection{Social Distancing}

One of the national responses that all countries take in the time of a pandemic is 'shielding' which is a self-imposed quarantine which people undergo to protect themselves from the spread of the pandemic. To reduce the exposure to the disease, social distancing is followed by controlling the level of contact with other individuals, which will help reduce human contact and eventually avoid transmission of the disease [22]. This significantly reduces the severity of the epidemic and it is useful at a time until vaccines are available. However, social distancing comes at a cost- including deprivation of freedom, financial resources, convenience, and also social contact [23]. People physically isolate themselves for some time during a pandemic or threat to psychosocial health. However, shielding can have an impact on psychological health, as suggested by Saathoff et al. [19]. Their research sheds light on how important it is for a nation to pay attention to the mental health of individuals, as a country that recovers from a pandemic without addressing the psychological side-effects can lead to rebuilding a country that might not have a spirit, vitality nor a sense of humanity.

\subsection{Chatbot}

A Chatbot is also called a 'ChatterBot'. It is a computer program based intelligent assistant that can respond to the person interacting through text messages and are powered by Artificial Intelligence (AI). A Chatbot communicates with the user based on the interaction model developed for it and it can create its system by learning common inputs and delivering answers, accordingly [24]. It is predicted that Chatbots would be used by people to engage with more than traditional websites or mobile phone applications in the future. Chatbots that use voice to chat are Amazon Alexa, Google Home, Siri, and Chatbots that use text-based inputs and outputs that can be accessed on platforms such as Messenger, Kik, and Slack. Users

can interact with the Chatbot using quick replies or texts and they are usable as well as easy for people to adapt and begin using.

Fig. 1 shows the screenshot the Chatbot as seen from the user's perspective. 


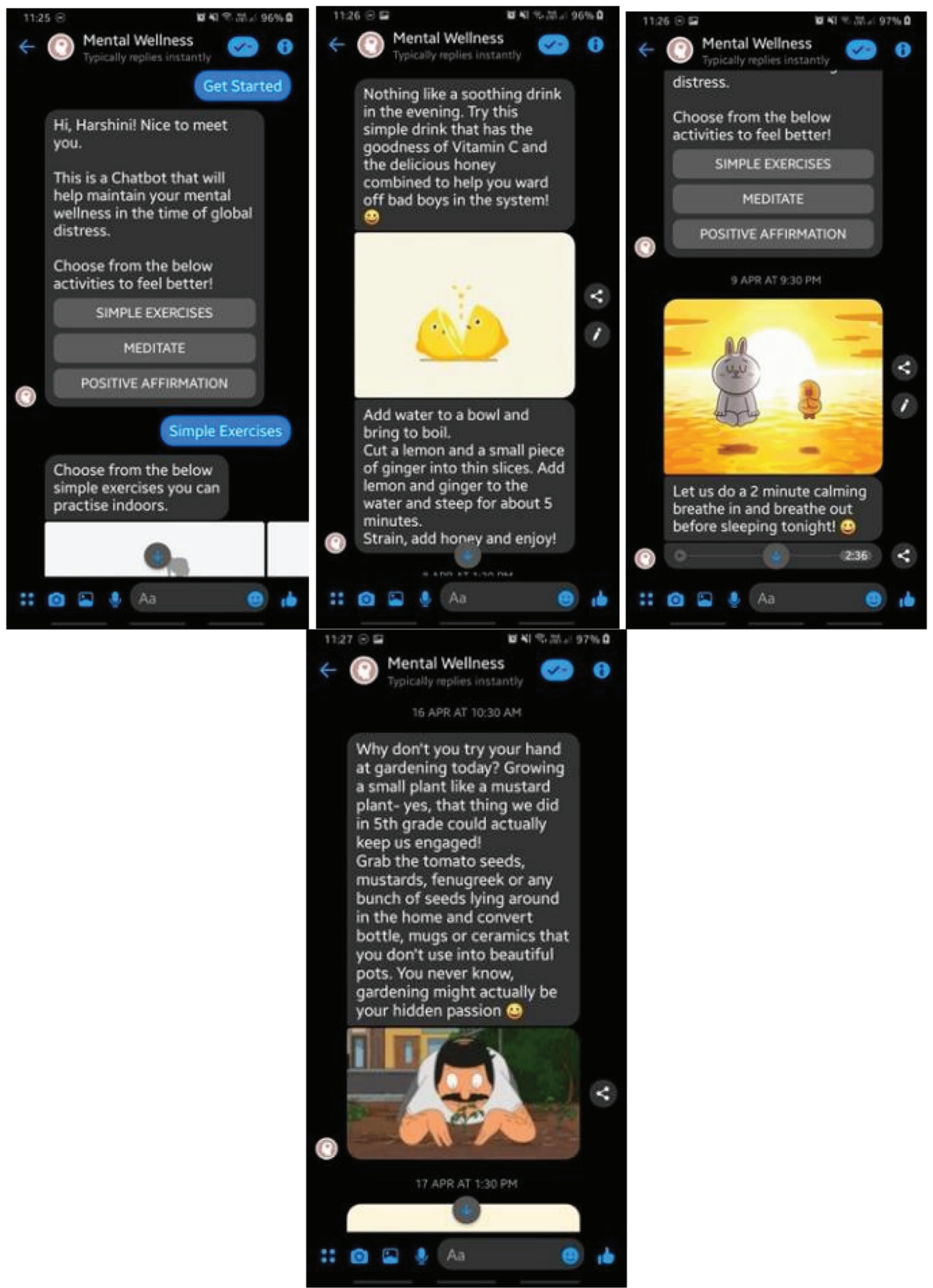

Figure 1: Screenshots from the Chatbot 
Chatbots are lately being created to help patients with a diagnosis of symptoms, to receive feedbacks instantly regarding basic health-related questions, and so on. Since Chatbots are trained to learn from previous interactions, it becomes helpful in mobile and e-health. Gradually, Chatbots are being increasingly used in the mental healthcare sphere. In this research, it is understood the number of users who signed up for the Chatbot only increased with time (Fig. 2). Chatbots like Woebot receive over two million messages in a week and a study conducted at Stanford University shows that people who used the Woebot Chatbot had significantly reduced symptoms of depression after two weeks of usage alone [25]. According to Singh [26], digital tools are now becoming feasible in reducing the treatment gap, as well as to make a psychiatric diagnosis and the treatment more affordable as well. Apart from this, the stigma associated with mental health and mental health illness has always been prevalent in India, e-mental health tools might be helpful. psychiatric disorders are a hindrance to seeking effective treatment. Younger adults do not have time and they are reluctant to seek a therapist for their mental health needs, and artificial intelligence (AI) could be a helping hand for them. Predicting the activities of people who are considering committing suicide and eventually the prevention of it, as well as identification of specific drugs that are used in psychiatry, as well as studying treatment responses, are some more ways AI is used in psychiatry in India.

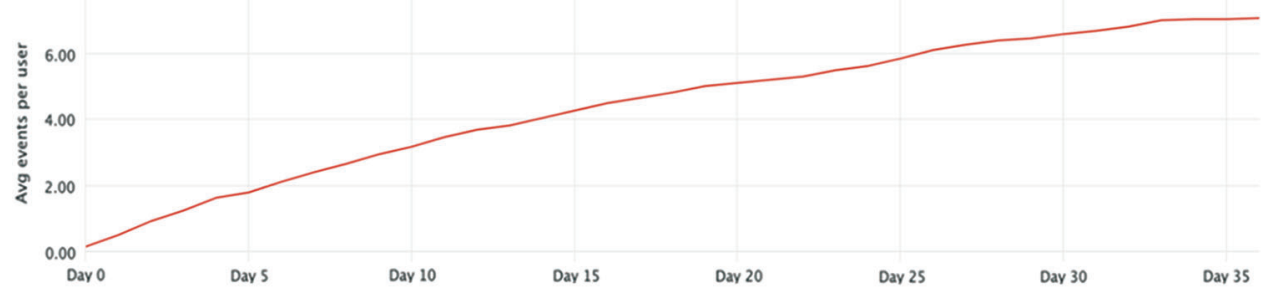

Figure 2: Increase in user engagement with time

When seen in the short term, digital tools like a Chatbot can help in reducing the psychological harms of being isolated. The aspect of receiving emotionally supportive messages from the tool has illustrated positive outcomes in certain contexts [25]. When designed and developed and put to use effectively, in the longer run, chatbots can help by reducing the psychological harms of isolation, trauma, and depression [27]. Maiden evidence shows that Chatbots may reduce mental health symptoms, but long-term consequences of using Chatbots are unclear and future research on this will prove to be worthy.

Digital interfaces are emerging as feasible alternatives for reducing mental health accessibility gap and it is helping make psychiatric diagnosis and treatment affordable as well. The stigma associated with psychiatric disorders is also a hindrance to seeking effective treatment. College students and young adults often lack the time or are hesitant to seek a therapist for their mental health needs. Artificial intelligence (AI) has made its venture into medical science many years ago, and the presence has been felt in mental health spheres also. Suicide prediction and prevention, identification of predictors for treatment response, and identifying which particular drug is best suited for a particular patient are some of the areas where AI is useful in psychiatry [18].

\section{Theoretical Framework}

The research employs the broaden and build theory of positive emotions [13]. Positive emotions, according to Fredrickson is defined as 'multicomponent response tendencies that unfold over relatively short periods'. Myers and Diener explain positive emotions as a key component in happiness or subjective well-being. Fredrickson also defines positive emotions as a sort of contentment and an element of love [28]. 
Personal resources that are collected and stored for a long time in states of positive emotions are very long-lasting. They can forego any other kind of emotional state that is acquired by the individual [29]. The chances of experiencing a positive emotion lead to an increase in the personal emotions of one which serve as reserves of positive emotions that can be drawn out and utilized at different points of emotional states. The broaden and build theory explains that on broadening positive emotions it allows individuals to build personal resources on their own at any emotional state, which could also be a negative emotional state. Data for training the bot was collected to provide basic activities to enhance the mood of the user and to feel positive.

If in case, negative emotions narrow down an individual's resources, positive emotions help in broadening this and thereby enabling individuals to experience these positive emotions acting as an antidote to the effects of gloom or negativity [30]. The positive thought-action experience can dismantle the hold of the negative emotion that has been accrued in the individual's mind, thus, nullifying the negative emotion. The nullifying act suggests that an individuals' mental health would improve their psychological wellbeing and in turn physical well-being by helping build positive emotions. Individuals can cope with negative emotions with the help of their positive thought-actions [31]. This argument is also supported by research conducted by Folkman and colleagues [15,32]. Blumenthal [33] comments that relaxation techniques trained by individuals help in reducing the pressure in blood and hypertension which is primarily done because of the effect of broadening that they capitalize on when practicing relaxation techniques. This technique falls in line with Fredrickson's [34] broaden and build theory. The research utilizes this theory for designing the messages that the Chatbot will deliver to the users. With the help of a professional psychiatrist, the messages that the Chatbot used to engage with the user were developed. The activities, cues, messages, and questions were developed in a manner that will help broaden positive emotions in the user. This method will help the user store the activities that induce positivity and utilize the activity or emotion benefitted from the activity at a later point in time where negative emotions seem to be narrowing down.

Research questions that will be covered in this research include; (RQ1) How effective it is to use a Chatbot to promote mental wellness and positivity during a pandemic and nation-wide lockdown situation? (RQ2) Can the Chatbot help individuals feel positive and promote self-care during a psychosocially distressful time?

\section{Methodology}

On 22nd March 2020 that the Prime Minister of India announced a 14-h test lockdown, called Janta curfew. Following this, the country was asked to observe Phase 1 of complete lockdown from 25 March 2020 till 14 April 2020 and phase 2 from 15 April to 03 May 2020. Since the phase 1 complete lockdown for about 21 days was announced on 24th March 2020, there was limited time available for the researchers to procure funding and to recruit participants for the study, hence the research was quickly kick-started to help the general public to cope with changes to their mental wellness that will arise because of something as new as a pandemic and lockdown. The Chatbot was gradually fed with the cues and it learned the inputs of the participants and it was developed into a full-fledged self-supported mental wellness Chatbot by the end of the 30-day study period.

For this one-group pre-test-post-test quasi-experimental research, the convenience sampling method was used to recruit 31 individuals who were not diagnosed for the coronavirus after receiving approval from the Ph.D. supervisor and Department of Media Sciences, Anna University's Ethics Committee. The participants were non-essential workers who may or may not be working from home. With the help of Instagram and Facebook advertising tools, campaigns were run to recruit participants from various work backgrounds, irrespective of any preferential gender selection between the age group of 23-45 years old were recruited. 
The participants were exposed to a three-step research module in the English language: Pre-test survey, intervention study, and a mixed-method post-test study.

After recruiting and signing the 31 participants who were willing to continue to be a part of the study, and after obtaining informed consent about the study and the usage of the data hence derived, the pre-test survey was sent to them. The pre-test consisted of a modified Kessler Psychological Distress Scale (K10) questionnaire, with a 5-point Likert scale answer option for each question. K10 is a measure of psychological distress that poses ten questions about emotional states. Each of these questions has a response scale of five levels [34], the results are illustrated in Table 1. After the completion of the pre-test survey, the participants were asked to use the Chatbot supported by Facebook messenger. The Chatbot is to be used during the lockdown period between 20 March 2020 and 20 April 2020.

Table 1: Questionnaire results summary

\begin{tabular}{lllllllllll}
\hline & $\mathrm{Q} 1$ & $\mathrm{Q} 2$ & $\mathrm{Q} 3$ & $\mathrm{Q} 4$ & $\mathrm{Q} 5$ & $\mathrm{Q} 6$ & $\mathrm{Q} 7$ & $\mathrm{Q} 8$ & $\mathrm{Q} 9$ & $\mathrm{Q} 10$ \\
\hline All the time & 2 & 1 & 3 & 4 & 3 & 1 & 3 & 0 & 2 & 0 \\
Once a while & 13 & 14 & 12 & 10 & 16 & 2 & 2 & 1 & 3 & 1 \\
Somewhat & 12 & 12 & 9 & 4 & 7 & 5 & 8 & 12 & 7 & 7 \\
Not much & 4 & 3 & 6 & 9 & 5 & 10 & 10 & 5 & 9 & 12 \\
Never & 0 & 1 & 1 & 4 & 0 & 13 & 8 & 13 & 10 & 11 \\
\hline
\end{tabular}

The Chatbot uses the English language to communicate with the audience and it begins with an induction message followed by re-engagement messages that were scheduled to be sent at different times during each day. The messages showed activity cues like exercises, meditation, reading, etc., as well as some recipes, music suggestions, and tips and tricks on how to keep up their mental wellness. With the help of a private mental health professional (MHP), the activities were identified, developed, and written. The MHP contributed to the development of the Chatbot by providing information on different activities that promote mental wellness and positivity that was suggested to the participants by the bot. The MHP does not hold an author credit as their support was sought twice over video call conferencing. The Chatbot was built using a third-party Chatbot development online tool- Chatfuel to deliver messages of positivity to the recruited participants. A message was sent each day for the participants to read or perform activities as mentioned.

Post the usage of the Chatbot, a mixed-method study using a survey and telephonic interview was implemented to study the impact of using the Chatbot. The participants were administered with a posttest questionnaire which consisted of altered questions from the pre-test also modified questions from K10 questionnaire, to measure the success and feasibility of the intervention. Participants were interviewed telephonically to understand the feedback. The users were also asked to provide suggestions that they might have for the future development of the Chatbot. The qualitative research analysis helped understand the impact of the Chatbot was on the users' mental wellness.

\section{Chatbot Design}

Conversation: The Chatbot-Mental Wellness is built based to promote positive emotions that can help cope with the negative emotions that loom over the COVID-19 pandemic. The conversational flow and script were developed at first, followed by the writing of trigger messages-which include simple activity triggers, quotes, positive news, and GIFs.

On-Boarding: The Chatbot opens with a welcome message that asks the user to choose an activity that will help them feel better. They can start by choosing one of the three-Meditation, Positive Affirmation, or 
Simple Exercises. The meditation cue will play an audio of three minutes where people can follow the guided meditation voice note and practice along with it. Positive Affirmation cue will throw in random one-liners that will provide a sense of positivity and up the self-confidence of the user. The simple exercises cue will show some illustrated images of simple exercises that the user can practice.

Features: The Chatbot will send one to two messages each day showing random simple messages like 'Go watch the sunset today evening and relax in the terrace', 'make an immunity-boosting drink', 'practice gardening', 'make a pandemic plan', 'you do not have to be productive-the quarantine is not a race' and so on.

Analysis: For this qualitative research that employs one group pre-test-post-test quasi-experimental design, the researcher used the modified K10 and USE questionnaires-Usability, Satisfaction and Ease of Use questionnaire that was devised by Lund [6] followed by a survey and in-depth telephonic interview to understand the usability and effectiveness of the Chatbot.

Privacy: The participants were asked to sign an agreement at the beginning of the study. The agreement indicated for what purpose the collected data would be used, by whom-the main processer who is the researcher in this case, and the sub-processor, the third-party analytics tool 'Yandex'. The participant is required to open the Chatbot link that has been provided and it will open up a messenger bubble using the Facebook Messenger app. Their information from Facebook is also taken into account by Yandex to provide a better understanding of the user and to develop a user persona.

\section{Results}

\subsection{Demographic}

The participants consisted of 18 female participants ( 58 percent) and 13 male participants ( 41.9 percent). 19 of the participants possessed a post-graduate degree and 12 of them had an undergraduate degree. Out of the 31 participants, 11 were self-employed, 5 were students, 3 in top management roles, 5 in mid-level roles in corporate organization, 3 were in financial roles, 3 in media, and one doctor. 21 of the participants were currently residing in Chennai, four were residing in Bengaluru, four in Mumbai, and two were residing in Hyderabad, one in Coimbatore, and one in Pune. The major share of the participants lied between the age group of 25 to 27 years old (19 participants). At the time of conducting the test, 10 of the participants were married, 7 in a relationship, and 14 of them were single. Out of all the participants, 19 of them were postgraduates and 10 of them graduate degree holders while 2 of the participants were high school graduates.

\subsection{Pre-Test Study}

Results from the 31 participants (18 female and 13 male) pre-test surveys show that they are nervous (32 percent) and stressed out about the prevailing global pandemic scenario. Participants have not tried to indulge themselves in any form of de-stressing activities like meditation, mindfulness, or even physical exercise to feel better or to feel positive before starting the experiment. The modified Kessler Psychological Distress Scale shows that many of the participants (42 percent) show minor symptoms of

distress however, 31 percent of the participants seem to show extreme symptoms of distress. Before the beginning of the intervention study, about 22.5 percent of the participants showed their interest in selfcare activities, physical exercise, or learn something new during the lockdown period. The rest of the participants (69 percent) had commented that they would continue to do their regular activities like working, cooking, and cleaning.

Two of the participants have a previous history of mental illness, being clinical depression, and anxiety. Both the participants showed reduced symptoms according to the reports from their psychologist and they have not been seeking treatment for the same in the past six months. The symptoms had significantly reduced over two years ago and there have been no relapses or higher risk in any of the participants. At 
the time of research, two of the participants had lost their job and both of them showed significantly higher symptoms of psychosocial distress and both of them do not have any history of mental illness.

The participants were very concerned about the speed at which the virus is spreading. More than half56.1 percent of the participants feel that the healthcare system in their respective regions is not effective enough to battle the spread of the pandemic. However, 51 percent of the participants feel hopeful that the situation is going to improve. Out of 31 participants, 35.4 percent of the participants anticipated that they would indulge in Yoga, meditation, or physical activities during the lockdown, 29 percent of the participants said that they would read and seven of them would cook. Five participants said they would probably, declutter their homes, or take online classes to learn something new or paint. However, 19 percent of the participants said they do not have anything to do during the lockdown period. It was also inferred from the pre-test study that the participants who were undergoing psychosocial distress are not interested in taking up any form of activity during the lockdown period.

Those who were married (29 percent) indicated lesser symptoms of psychosocial distress. Out of the 10 participants who indicated higher symptoms of distress, 9 of them are single. Being single or lockeddown alone could be a trigger for increased signs of distress.

\subsection{Chatbot}

On launching the Chatbot after filling the pre-test survey, the subscribers will be shown a welcome message with three call-to-action (CTA) buttons- Simple exercises, meditate, and positive affirmations. On tapping any of the activity the user will be taken to the exercise cue-a guided meditation voice notes for meditation, a set of positive messages for positive self-affirmation, and simple exercise illustration based images to help the user do the same. After introducing them to the basic activities, the Chatbot sent re-engagement messages every day to the subscribers triggering them to do one activity a day. Messages included-a trigger for watching the sunset, calling a friend, taking a walk in the terrace, gardening, etc.

\subsection{Usage of the Chatbot}

The participants gradually adapted to using the Chatbot and they show a steady increase in the engagement. The number of inputs received from the participants increased with time.

The average session length was $7 \mathrm{~s}$. The majority of the users' sessions lasted for $1-9 \mathrm{~s}(87.2$ percent), followed by $10-29 \mathrm{~s}$ ( 4.26 percent) and 30-59 s (2.1 percent). Participants who used it for more than 4 min to 19 min constitute about (6.3 percent).

Out of the 31 recruited participants, in between the study period of 16 March 2020 to 19 April 2020, there were two drop-outs. One of the participants 'blocked' the Chatbot, thereby ending the subscription to the bot on 23 March 2020 and another participant on 16 April 2020. There was a total of 31 active users (including the researcher), with one dropping out in March 2020 and the other in April 2020. However, the participants who blocked incoming messages from the Chatbot did take the post-test study as well as provided their inputs about the Chatbot.

\subsection{Post-Test}

All the 31 respondents used the Chatbot, with two dropouts during the 30-day study period. Meditation (64.5 percent) and positive affirmations (67.7 percent) seem to be the most popular task among the users, followed by exercise (19.4 percent), recipes (9.7 percent), and others (9.7 percent). Most of the participants (64.4 percent) opened and utilized the Chatbot even without the push notification. The participants opened the Chatbot to exercise positive affirmations ( 79.2 percent). About 83.9 percent of the participants seem to have found the Chatbot extremely useful during the lockdown period. One 
participant did not find the Chatbot useful, and four participants are undecided if they found the Chatbot useful.

The Chatbot's interface is very similar to smartphone messaging apps like Messenger, WhatApp, Telegram, etc., making it easy for users to learn. 61.3 percent of the users found the Chatbot easy to use and 93.3 percent of the audience think the Chatbot helped them be more effective. The user was allowed to interact freely with the Chatbot in their own time and need. After using the Chatbot, the user was asked to fill a questionnaire with four sections- basic Chatbot related questions, modified Kessler scare of psychosocial distress questionnaire, Chatbot usage, and Chatbot usability. After receiving the responses, the researcher conducted a telephonic interview with the user to understand and record their experiences and level of satisfaction on using the Chatbot.

The interview was structured around three questions:

1. How did the Chatbot help you during the lockdown period?

2. What is your biggest takeaway on using the Chatbot?

3. Do you have any feedback or suggestions to improve this Chatbot, when designing this for the public to be used during a time of global distress?

4. What else can we add to the Chatbot to promote mental wellness at a time of Pandemic?

The Likert scale values were scored out of 50. The lower the score indicated better mental health and reduced psychosocial distress. The Chabot has helped elevate the mental wellness of the participants. 19 of the participants have secured scores greater than 20 and below 30 indicating an increase from 15 participants from the pre-test Kessler scale scores (Fig. 3). This simply denotes how the Chatbot has uplifted the mental health of almost all the participants who took part in the research.

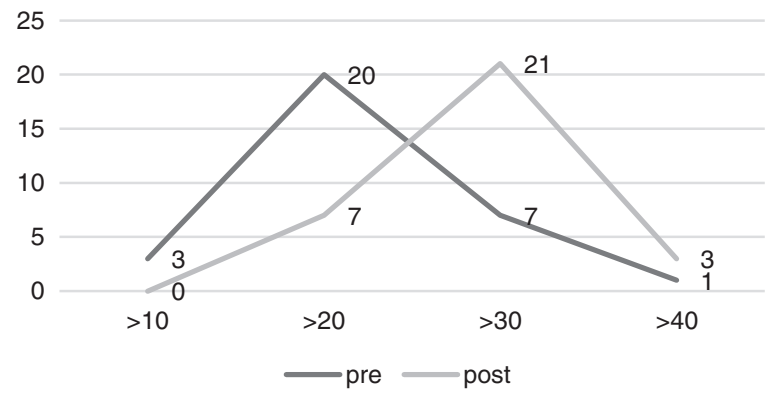

Figure 3: Comparative line graph of the modified kessler scale in both pre-and post test

Table 2 explains the positives, negatives, and suggestions provided by the user over the telephonic interview. The Chatbot has helped users understand that the positivity is within themselves. About four of the participants feel that the Chatbot is more like a friend that is overlooking them during times of social distress. The Chatbot's messages provided them with positivity and hopefulness when things seem bleak in the outside world. Participantd expressed that 'positive affirmations' was a new task that they learned on using the Chatbot. Most of the participants provided a positive opinion about the Chatbot and that it kept them engaged by providing various new cues each day.

One of the participants who dropped out by blocking the Chatbot on 23 March 2020, noted that the messages from the Chatbot was 'spam' and neither were they interested nor was the Chatbot messages relevant to. The second dropout expressed their liking towards the Chatbot, however, they had learned the techniques and were not keen on utilizing them anymore as they had gotten into a routine during the lockdown. A few comments from other users showed that they felt the Chatbot's re-engagement 
messages could have been more tailor-fit to meet the needs of the participants rather than providing generic tips. Three other participants felt that the Chatbot should have asked their current state of mind and prescribed activities and cues according to the mental state they are in. Two of the participants explained that the Chatbot should be more conversational; more two-way communication can be incorporated rather than providing cues each day. Participants suggested that the Chatbot could have been made into a smartphone application rather than a Facebook supported Chatbot. One of the participants suggested that the Chatbot could have been an all-rounder by providing COVID-19 updates. Rather than providing only positive news, the app could have given physical health measures and statistics relating to the pandemic as well.

Table 2: Chatbot interview topic analysis

\begin{tabular}{|c|c|c|}
\hline Positivity is within me & I had no time & Make it an app \\
\hline 5 & 2 & 2 \\
\hline $\begin{array}{l}\text { Kept me engaged/activities were } \\
\text { very helpful }\end{array}$ & It spammed me & $\begin{array}{l}\text { Make it a regular and not just } \\
\text { for the pandemic }\end{array}$ \\
\hline 3 & 2 & 1 \\
\hline $\begin{array}{l}\text { Had someone to look out for } \\
\text { me/virtual friend }\end{array}$ & Make it more conversational & $\begin{array}{l}\text { Could have provided } \\
\text { COVID-19 updates }\end{array}$ \\
\hline 4 & 3 & 1 \\
\hline $\begin{array}{l}\text { Something good during bad } \\
\text { times/destressing/hopeful }\end{array}$ & $\begin{array}{l}\text { It could have been an open space to } \\
\text { share my thoughts/feelings }\end{array}$ & Add podcasts \\
\hline 4 & 1 & 1 \\
\hline Learned something new & Make it more tailored to audience needs & \\
\hline 3 & 3 & \\
\hline I took care of myself & Need more ways to destress & \\
\hline 2 & 1 & \\
\hline Lifted my spirits & Remove physical exercise & \\
\hline 2 & 2 & \\
\hline
\end{tabular}

One of the participants even expressed that the Chatbot helped them sleep better because of the short meditation audio clip that they listened to each night. About 32.2 percent of the participants found exercises to be stressful as they are not physically active. However, they preferred meditating and practicing positive affirmations over physical exercise. 48.3 percent of the participants expressed that they would continue to meditate and 22.5 percent said they would continue exercising positive affirmations in their daily lives. The most popular activities that the participants used were positive affirmations (21 taps) and meditation (18 taps), followed by exercise (13 taps). The Chabot has helped 86.7 percent of the participants feel more positive and 70 percent of them who set a goal to be achieved during the lockdown period said the Chatbot helped them achieve them.

The Chatbot developer's backend tool allows us to gain an insight into the usage of the Chatbot by providing data of live users, most clicked buttons, most inputs received, and session lengths. Positive affirmations and meditate has been the most used activities by the users. The week between 06 April 2020 to 12 April 2020, saw the highest number of re-engagement and the number of sessions the participants had with the Chatbot increased between 13 April 2020 and 19 April 2020. 


\section{Limitations}

One of the major limitations in terms of recruitment was the inability to assess the willingness of the participant online. Out of all the participants who signed up to take the study in reply to the social media ad campaign which was 75 individuals, only 31 of the participants continued to show willingness when the pre-test study forms were sent. As the researcher wanted to begin the study as soon as the lockdown was imposed to reduce the intensity in mental illness that may arise among the general public and to quickly provide the intervention, the ad campaign to recruit the participants was run only for 7 days-with an ad budget of 1000 INR from the personal social media handles of the researcher.

The time to develop the Chatbot was limited as the Chatbot had to be dispatched for use immediately after the lockdown was announced. However, the functionality and messaging were slowly developed with time. The researcher could not meet the participants to conduct interviews or to administer the questionnaire because of the lockdown.

As the Chatbot was powered by a third-party tool available on the internet called 'Chatfuel', some of the features in terms of analysis, identification of participants was limited. The research used other tools like 'Yandex App Metrics' tools to acquire detailed reports on the session length, dropouts, and user persona. However, this was also a paid tool that the researcher couldn't purchase due to financial limitations.

\section{Discussion}

COVID-19 has not only affected people physically, but both psychologically, and especially in a developing country, there has also been a huge social impact of the pandemic. The psychosocial distress levels shoot up during such incomprehensible times. However, the number of people who sought mental health support has increased. Since India's response to the pandemic was to impose a lockdown to limit people from going out except for essentials, hotlines, social media accounts, websites, and webinars were set up by both the Government and private entities to help people cope up with the mental stress and anxiety induced by the lockdown and pandemic. Since the social needs in India like transportation for the stranded migrants, food for all, and housing for the destitute was higher, the capability to accommodate the mental health needs of the general public seemed not only herculean but took a backseat when putting on par with basic social living needs of the people.

Certain private platforms and other NGOs set up their hotlines, websites, developed mobile phone applications, and conducted live sessions on social media or webinars to help people cope up with the staggering changes in their mental health. Many of these were effective and the fact that there is one in three people owning a phone makes the reach of these hotlines even more extensive thereby helping people remain mentally well. According to the National Mental Health Survey (2016), there are a higher number of patients suffering from mental illness in urban locations. This illustrates the availability of knowledge and smartphones within their reach. The Ministry of Health and Family Welfare created online resources that could help people cope up with mental illness arising from the pandemic, and many NGOs and private psychology practitioners took webinars, lectures, and live sessions using social media platforms to educate people. However, there was no such Chatbot that helped the people not affected by the coronavirus deal with their mental wellbeing. Hence, this research helped develop a smartphone supported Chatbot to promote mental wellness among people during the distressful times of COVID-19 in India.

The Chabot was developed using the broaden and build theory of positive emotions. The Chatbot would send across one or more re-engagement messages to the participants of the study to help them with basic coping mechanisms like positive affirmations tasks, meditation, doodling, journaling, etc. Since the broaden and build theory works towards cultivating positive emotions over negative ones, the techniques used by the Chatbot might help reduce symptoms of anxiety, sadness, and loneliness in the users. The 
qualitative research employed a one-group pre-test-post-test quasi-experimental research design approach, assigning 31 participants between the age group of 23-45 years old. The mixed-method post-test study using questionnaires and telephonic interviews helped understand the impact of using the Chatbot in improving the symptoms of psychosocial distress which was measured using a modified Kessler Scale Scoring.

From the research, it has been understood that a lot of the general public who are not affected by the virus, are undergoing symptoms of psychosocial distress. They are mostly worried about the speed at which the virus is spreading and the lack of adequate healthcare facilities in India to support the intensity of the pandemic. There is also an influx of 'infodemic', where people are receiving a multitude of information from social media platforms thereby increasing the stress in them. Although many show symptoms of distress, they are unable to understand how to seek help to feel better. Hence, the Chatbot seemed like the right way for them to feel better during these distressful times.

In terms of usability, the Chatbot mimicked the interface of WhatsApp, Messenger, WeChat, Viber, etc., thereby making it very easy for the users to adopt this tool in their everyday life. Since the Chatbot was supported by Facebook messenger, anyone who has access to a Facebook account can utilize the Chatbot. This reduces the time taken to learn new technology and provides immediate results as users are already familiar with the usability of such apps.

The majority of the participants found the Chatbot very useful and that it helped improve their mental wellness and reduce symptoms of distress by keeping them engaged and providing them with newer ways to feel positive. The majority of the participants helped achieve a better mood thanks to the Chatbot. Two participants dropped out of the research by blocking the Chatbot. One said the messages were spamming them and the other mentioned that they learned the techniques and were incorporating them in their lives and did not require any further triggers. However, both the participants who dropped out did feel it brought in some kind of awareness about their mental wellness during the pandemic.

One of the participants explained how the Chatbot's meditation audio clips helped them receive sound sleep, especially at times when they could not fall asleep because of the 'messed up' routine during the lockdown. Not all participants want to indulge in physical exercises to feel better mentally. However, they prefer simple activities like positive affirmations to elevate their mood. Meditation and positive affirmations seem to be the most liked task to do. Many were not aware of an activity called positive affirmations. They are hoping to continue to use meditation and positive affirmations tasks in their everyday lives even after the research period. Learning new ways to keep themselves occupied and newer techniques to feel mentally fit will help participants engage in them and to utilize technological tools like a Chatbot or an app better.

Users started slowly getting acquainted with the Chatbot and they re-engaged with the app more during the fourth week, and the number of sessions seems to have increased in the fifth week of intervention. This indicates that people take time to engage with a new intervention when they are distressed. The Chatbot intervention helped all six of the individuals who showed increased symptoms of psychosocial distress (as per the K10) by significantly reducing symptoms by the end of the intervention, as indicated by the post-test. One of the key suggestions from the users was that the messages should be more tailor-made for each of the mental issues rather than have one common message for all ailments. Some suggested, there could also be Coronavirus updates through the Chatbot, which would help everyone check the bot every day and read all the messages.

From the interview that was conducted, it was understood that the majority of the participants strongly feel that positivity is within themselves-which was the main focus of building the Chatbot based on the broaden and build theory. The Chatbot also provided the participants with something to feel hopeful for, or do something good to keep themselves happy and engaged. Some of the participants also feel they 
learned something new and the Chatbot helped remind them to take care of themselves and feel uplifted. Two participants echoed on how the Chatbot could be made into and app and one more participant resonated on how the Chatbot could be created for everyday use and not just at times of disasters, social threats, or pandemics.

Broaden and build theory helps in creating positive emotions that can help forego negative emotions when they arise. In line with the theory, many participants commented that they would incorporate basic activities inspired by the Chatbot in their everyday lives even after the research period or pandemic. Since this research was subject to a few limitations like the lack of time to strategize and lack of funding, which was slowly acquired and input as and when needed, incorporating these can help in future studies about digital interventions for promoting mental wellness during disasters or pandemics.

\section{Future Studies}

Future research can be done on creating a full-fledged mobile phone application to promote all-round mental wellness for the individuals not affected by the coronavirus in the times of disasters or pandemics. Chatbots developed for mental health wellbeing need to be more conversational-making them friendlier and they would like to speak with someone when undergoing psychosocial distress. A pandemic is something new that people experience- they are scared and shocked at the speed at which diseases spread and especially when they are forced to remain indoors to contain the spread. Using digital tools like Chatbots or Apps to promote mental wellness can help individuals not be affected by the coronavirus cope up with a growing pandemic in keeping themselves mentally fit.

With the increase in the need for mental health support not only at times of distress but also during normal times, and keeping in mind the lack of availability of mental health professionals (National Mental Health Survey, 2020) in India, using a digital tool like a Chatbot can help one professional reach out to a lot more people. The availability of a digital tool to bridge the gap between a mental health professional and the patient can provide far-reaching results. Although Chatbots cannot replace the practitioner, the bot can help as an initial analysis tool for psychotherapists to provide mental healthcare accordingly.

Chatbots can also be created with simple self-care tips and tricks for people to incorporate in their everyday lives. Digital tools and artificial intelligence are evolving day by day, and Chatbots being an effective tool, they can be utilized by both the Government and private professionals thereby making mental healthcare more accessible than ever. Cultural considerations shall also be made, for example, the month of June was the month of Ramadan, the future apps or Chatbots could deliver mental health and wellness support for those fasting during this period.

Future studies can be conducted by received increased funding and resources to implement a holistic Chatbot to promote mental wellness by using the broaden and build theory which emphasizes the availability of positive emotions and utilization of it in the time of need by the individual. Although the results seem evident of the change a digital intervention can bring about in the mental wellness of the general public, the use of a control group can also help demonstrate the effectiveness of using a Chatbot.

\section{Conclusions}

The Chatbot was designed to help promote positivity and better mental wellbeing among the individuals not affected by the coronavirus who were self-isolating during the lockdown period in India. Using the broaden-and-build theory, promoting positivity can help forego negative emotions like stress, anxiety, and fear. Overall, the experimental research proved successful as everyone used the Chatbot and the majority of positive reviews indicate the usefulness of the tool during a time of global distress, especially for the individuals not affected by the coronavirus who are forced to follow social distancing and remain indoors and are psychosocially distressed. The Chatbot was used extensively by the users and the re-engagement 
of users only increased with time. The Chatbot was well received and promoted positivity to the majority users, however, some users suggested that the Chatbot could be more focussed depending on the mental issue undergone by the user and a more conversational feature could help improve the Chatbot. Larger sample size and funding provided for the research could also help in including more features and a deeper understanding of the incorporation of such a Chatbot by governmental organizations.

Acknowledgement: The researcher acknowledges the inputs provided by the various psychoherapists and mental health practitioners for their inputs on developing the Chatbot.

Funding Statement: The authors received no specific funding for this study.

Conflicts of Interest: The authors declare that they have no conflicts of interest to report regarding the present study.

\section{References}

1. Lu, H., Stratton, C. W., Tang, Y. W. (2020). Outbreak of pneumonia of unknown etiology in Wuhan, China: The mystery and the miracle. Journal of Medical Virology, 92(4), 401. (in Chinese). DOI 10.1002/jmv.25678.

2. Iancu, I., Strous, R., Poreh, A., Kotler, M., Chelben, Y. (2005). Psychiatric inpatients' reactions to the SARS epidemic: An Israeli survey. The Israel Journal of Psychiatry and Related Sciences, 42(4), 258.

3. Cameron, G., Cameron, D., Megaw, G., Bond, R., Mulvenna, M. et al. (2018). Assessing the usability of a chatbot for mental health care. International Conference on Internet Science, pp. 121-132. Springer, Cham.

4. Chinese Association for Disaster and Emergency Rescue Medicine (2020). National crisis intervention platform for New Coronavirus Pneumonia (in Chinese). http://wwwcastorgen/art/2020/2/3/art_80_109851.html.

5. Li, W., Yang, Y., Liu, Z. H., Zhao, Y. J., Zhang, Q. et al. (2020). Progression of mental health services during the COVID-19 outbreak in China. International Journal of Biological Sciences, 16(10), 1732-1738. DOI 10.7150/ ijbs. 45120 .

6. Lund, A. M. (2001). Measuring usability with the USE questionnaire. STC Usability SIG Newsletter, 8(2), 3-6.

7. Huang, C., Wang, Y., Li, X., Ren, L., Zhao, J. et al. (2020). Clinical features of patients infected with 2019 novel coronavirus in Wuhan, China. Lancet, 395, 497-506. DOI 10.1016/S0140-6736(20)30183-5.

8. Ramalingaswami, V. (2001). Psychological effects of the 1994 plague outbreak in surat, India. Military Medicine, 166(2), 29-30. DOI 10.1093/milmed/166.suppl_2.29.

9. Manderscheid, R. W. (2007). Preparing for pandemic avian influenza: Ensuring mental health services and mitigating panic. Archives of Psychiatric Nursing, 21(1), 64-67. DOI 10.1016/j.apnu.2006.10.003.

10. Chan, A. O., Huak, C. Y. (2004). Psychological impact of the 2003 severe acute respiratory syndrome outbreak on health care workers in a medium size regional general hospital in Singapore. Occupational Medicine (Land), 54(3), 190-196. DOI 10.1093/occmed/kqh027.

11. Sim, K., Chua, H. C. (2004). The psychological impact of SARS: A matter of heart and mind. Canadian Medical Association Journal, 170, 811-812. DOI 10.1503/cmaj.1032003.

12. Ursano, R. J. (2005). Preparedness for SARS, influenza, and bioterrorism. Psychiatric Services, 56(7). DOI 10.1176/appi.ps.56.1.7.

13. Gao, J., Zheng, P., Jia, Y., Chen, H., Mao, Y. et al. (2020). Mental health problems and social media exposure during COVID-19 outbreak. https://ssrn.com/abstract $=3541120$.

14. Chan, S. S. C., So, W. K. W., Wong, D. C. N., Lee, A. C. K., Tiwari, A. (2007). Improving older adults' knowledge and practice of preventive measures through a telephone health education during the SARS epidemic in Hong Kong: A pilot study. International Journal of Nursing Studies, 44, 1120-1127. DOI 10.1016/j. ijnurstu.2006.04.019. 
15. Kumar, N., Gupta, N., Naval, C., Adhikari, T., Tiwari, M. N. N. et al. (2018). Prevalence of tools of digital communications among population: A ground study in tribal, rural and urban slum. Journal of International Medical Science Academics, 31(2), 1-6.

16. Psychological crisis intervention for 'Novel pneumonia' (in Chinese) (2020). Wuhan Mental Health Center. http:// dy163com/v2/article/detail/F3URP4L805346KFD.html.

17. Chinese Society of Psychiatry (2020). Online lessons of psychological intervention during the New coronavirus pneumonia outbreak (in Chinese).

18. Guidelines for psychological assistance hotlines during 2019-nCoV pneumonia epidemic (2020). National Health Commission of China.

19. Saathoff, G., Everly, G. S. Jr. (2002). Psychological challenges of bioterror: Containing contagion. Introduction to Emerging Mental Health, 4, 245-252.

20. Chinese Psychological Society (2020). Psychological responses in the fight against New crown pneumonia (in Chinese). https://mpweixinqqcom/s/-JBOVmi8jDqtUhNIh7Pow.

21. Kessler, R. C., Barker, P. R., Colpe, L. J., Epstein, J. F., Gfroerer, J. C. et al. (2003). Screening for serious mental illness in the general population. Archives of General Psychiatry, 60(2), 184-189. DOI 10.1001/ archpsyc.60.2.184.

22. Del, V. S., Hethcote, H., Hyman, J. M., Castillo-Chavez, C., Math, B. (2005). Effects of behavioral changes in a smallpox attack model. PubMed, 195(2), 228-251. DOI 10.1016/j.mbs.2005.03.006.

23. Reluga, T. C. (2010). Game theory of social distancing in response to an epidemic. PLoS Computational Biology, 6(5), e1000793. DOI 10.1371/journal.pcbi.1000793.

24. Oh, K. J., Lee, D., Ko, B., Choi, H. J. (2017). A chatbot for psychiatric counseling in mental healthcare service based on emotional dialogue analysis and sentence generation. IEEE International Conference on Mobile Data Management, pp. 371-375. DOI 10.1109/MDM.2017.64.

25. Ho, A., Hancock, J., Miner, A. S. (2018). Psychological, relational, and emotional effects of self-disclosure after conversations with a chatbot. Journal of Communication, 68(4), 712. DOI 10.1093/joc/jqy026.

26. Singh, O. (2019). Chatbots in psychiatry: Can treatment gap be lessened for psychiatric disorders in India. Indian Journal of Psychiatry, 61(3), 225-225. DOI 10.4103/0019-5545.258323.

27. Brooks, S. K., Webster, R. K., Smith, L. E., Woodland, L., Wessely, S. et al. (2020). The psychological impact of quarantine and how to reduce it: Rapid review of the evidence. The Lancet. DOI 10.1016/S0140-6736(20)30460-8.

28. Huremović, D. (2019). A mental health response to infection outbreak. In: Psychiatry of pandemics, pp. 95-118. Basel: Springer Nature Switzerland AG.

29. Lazarus, R. S., Kanner, A. D., Folkman, S. (1980). Emotions: A cognitive-phenomenological analysis. In: Theories of emotion, pp. 189-217. Academic Press. DOI 10.1016/B978-0-12-558701-3.50014-4.

30. Fredrickson, B. L., Levenson, R. W. (1998). Positive emotions speed recovery from the cardiovascular sequelae of negative emotions. Cognition and Emotion, 12, 191-220. DOI 10.1080/026999398379718.

31. Fredrickson, B. L. (2000). Cultivating positive emotions to optimize health and well-being. Prevention and Treatment. http://www.journals.apa.org/prevention/volume3/pre0030001a.html.

32. Folkman, S. (1997). Positive psychological states and coping with severe stress. Social Science Medicine, 45, 1207-1221. DOI 10.1016/S0277-9536(97)00040-3.

33. Blumenthal, J. A. (1985). Relaxation therapy, biofeedback, and behavioural medicine. Psychotherapy: Theory, Research, Practice, Training, 22(3), 516. DOI 10.1037/h0085536.

34. Fredrickson, B. L. (2001). The role of positive emotions in positive psychology: The broaden-and-build theory of positive emotions. American Psychologist, 56(3), 218. DOI 10.1037/0003-066X.56.3.218. 